\title{
MS14-P25 | A NEW POLYMORPH OF TRISODIUM HEXACHLORORHODATE
}

Etter, Martin (Deutsches Elektronen-Synchrotron, Hamburg, GER)

Several trisodium hexachlorometalates $\left(\mathrm{Na}_{3} \mathrm{MCl}_{6}\right.$ with e.g. $\mathrm{M}=\mathrm{Ir}$, Rh) are highly hygroscopic and often exhibit simultaneously multiple hydrated phases at ambient conditions. In order to determine the different crystal structures of these hydrated compounds, in situ powder X-ray diffraction experiments are performed, where the temperature is slowly increased and the evaporation of water leads to less hydrated compounds with lower water content until finally the anhydrous phase is formed. Usually, during this process the different hydrated phases become separated, which makes it easier to distinguish them and to start crystal structure solution attempts.

However, after performing a couple of in situ powder X-ray diffraction measurements on $\mathrm{Na}_{3} \mathrm{RhCl}_{6}$ and $\mathrm{Na}_{3} \mathrm{IrCl}_{6}$ it became clear, that also at elevated temperatures mixtures of hydrated phases are always present until the anhydrous compound is formed. Therefore, only the crystal structures of the anhydrous phases of $\mathrm{Na}_{3} \mathrm{RhCl}_{6}$ and $\mathrm{Na}_{3} \mathrm{IrCl}{ }_{6}$ have been determined so far.

Intriguingly, in some of the heating experiments on $\mathrm{Na}_{3} \mathrm{RhCl}_{6}$ a second phase coexisted at elevated temperatures together with the anhydrous phase until it was transformed fully into the known anhydrous $\mathrm{Na}_{3} \mathrm{RhCl}_{6}$ compound. This phase was determined to be a polymorph of $\mathrm{Na}_{3} \mathrm{RhCl}_{6}$ which surprisingly possesses the same space group as the stable anhydrous $\mathrm{Na}_{3} \mathrm{RhCl}_{6}$ phase, although lattice parameters and motif are different.

In this presentation structural details of the dehydrated $\mathrm{Na}_{3} \mathrm{RhCl}_{6}$ polymorph will be given as well as an overview to the so far performed in situ powder X-ray diffraction experiments on the hydrated phases. 